\title{
Business Time and New Credit Risk Models
}

\author{
Elisa Luciano \\ Università di Torino, ICER \& Collegio Carlo Alberto \\ Convegno Economia e Incertezza, Trieste, 23 Ottobre \\ 2009
}

\begin{abstract}
This paper examines a new model of credit risk measurement, the Variance Gamma- Merton one, which seems to be adequate for describing single default occurrence and default correlation in turbulent times. It is based on the notion of business time. Business time runs faster than calendar time when the market is very active and a lot of information arrives; it runs at a slower pace than calendar time when few information arrives. We report a calibration to USA spread data, which shows the accurateness of the model at the single default level; we also compare the perfeormance wrt a traditional structural model at the joint default level.
\end{abstract}

\section{Introduction}

This paper examines a new model of credit risk measurement, the Variance Gamma- Merton one, which seems to be adequate for describing single default occurrence and default correlation in turbulent times, such as the current, crisis or after-crisis ones. It is based on the notion of business time. Business time runs faster than calendar time when the market is very active and a lot of information 
arrives; it runs at a slower pace than calendar time when few information arrives. We report a calibration to USA spread data, which shows the accurateness of the model at the single default level; we also compare the performance with respect to a traditional structural model at the joint default level.

The structure of the paper is as follows: section 2 resumes the basic pre-crisis structural model of credit risk, both for single and joint defaults. In the multiple default case it specifies both the extended and simplified - or factor copula - version. It concludes with an overview of its shortcomings. Section 3 sets up our alternative, business-time credit model, both for single and joint default occurrences. Section 4summarizes its calibration to USA spread data. Section 5 presents the calibration to joint defaults. The conclusions follow.

\section{The pre-crisis base model of credit risk}

\subsection{Single defaults: Merton}

The basic pre-crisis structural model of credit risk is inspired by the seminal paper by Merton (1974). Consider a firm $i$ which has a single zero coupon debt. It expires at maturity $t$ and has face value $K_{i}>0$. Default of firm $i$ occurs - at debt maturity - if the firm's asset value $V_{i}(t)$ falls at or below the liability one, $K_{i}(t)$. The default probability at maturity $t$ is

$$
F_{i}(t)=P\left(V_{i}(t) \leq K_{i}(t)\right)
$$

while the distribution of the time to default, $\tau_{i}$, is

$$
\tau_{i}=\left\{\begin{array}{cc}
t & P\left(V_{i}(t) \leq K_{i}(t)\right) \\
+\infty & P\left(V_{i}(t)>K_{i}(t)\right)
\end{array}\right.
$$

Merton assumes that $\log$ asset prices $\left(\ln V_{i}\right)$ or asset returns are normal, i.e. Gaussian. Equivalently, he assumes that the asset value 
$V_{i}$ follows a geometric Brownian motion. Under his assumptions the (marginal) default probability of firm $i$ is

$$
F_{i}(t)=\Phi\left(-d_{2 i}(t)\right)
$$

where $\Phi$ is the (univariate) standard normal distribution,

$$
d_{2 i}:=\frac{\ln \left(V_{i}(0) / K_{i}(t)\right)+\left(\mu_{i}-\sigma_{i}^{2} / 2\right) t}{\sigma_{i} \sqrt{t}}
$$

$\mu_{i}$ and $\sigma_{i}$ are respectively the instantaneous mean return on assets ( $r$ under the risk neutral measure) and its standard deviation.

\subsection{Joint defaults}

For simplicity, we consider the bivariate case to start with. Two firms (or names), denoted as $i=1,2$, exist. In Merton's model, if one assumes that $\log$ assets are jointly normally distributed ${ }^{1}$, with correlation coefficient $\rho$, the joint default probability of two names with common expiry of debt is

$$
F(t)=P\left(V_{1}(t) \leq K_{1}(t), V_{2}(t) \leq K_{2}(t)\right)=\Phi_{\rho}\left(-d_{21}(t),-d_{22}(t)\right)
$$

where $\Phi_{\rho}$ is the (bivariate) standard normal distribution with correlation coefficient $\rho$.

Using the fact that $-d_{2 i}(t)=\Phi^{-1}\left(F_{i}(t)\right)$, the joint default probability can be represented as:

$$
F(t)=\Phi_{\rho}\left(\Phi^{-1}\left(F_{1}(t)\right), \Phi^{-1}\left(F_{2}(t)\right)\right)
$$

This is the so called copula representation of the joint probability. It is made possible by Sklar's theorem: for details see for instance $^{2}$ Nelsen (1998), or Cherubini, Luciano, Vecchiato (2004).

\footnotetext{
${ }^{1}$ As is known, joint normality is a further assumption wrt marginal normality.

${ }^{2}$ Both these references not only introduce the notion of copula and Sklar's theorem, but also the Gaussian and Students' copulas, to be used below. Nelsen is the seminal book in the field, Chr.... is mainly focused on Finance applications.
} 
The copula which we have obtained is the so-called (bidimensional)

Gaussian copula, defined as

$$
C_{G}\left(u_{1}, u_{2}\right):=\Phi_{\rho}\left(\Phi^{-1}\left(u_{1}\right), \Phi^{-1}\left(u_{2}\right)\right)
$$

Analogously, for the $n$ names, the joint default probability is

$$
F(t)=P\left(V_{1}(t) \leq K_{1}(t), \ldots . V_{n}(t) \leq K_{n}(t)\right)=\Phi_{R}\left(-d_{21}(t), \ldots,-d_{2 n}(t)\right)
$$

which can be written using the corresponding $n$ dimensional Gaussian copula, with correlation matrix $R$ :

$$
F(t)=\Phi_{R}\left(\Phi^{-1}\left(F_{1}(t)\right), \ldots, \Phi^{-1}\left(F_{n}(t)\right)\right)
$$

Instead of joint normality, one can assume a Student t copula among asset values:

$$
C_{t}\left(u_{1}, u_{2}, . ., u_{n}\right):=t_{R, \xi}\left(t_{\xi}^{-1}\left(u_{1}\right), t_{\xi}^{-1}\left(u_{2}\right), \ldots, t_{\xi}^{-1}\left(u_{n}\right)\right)
$$

where $t_{R, \xi}$ is the $n$-dimensional Student's t distribution of parameter $\xi$, while $t_{\xi}$ is the uni-dimensional Student's t distribution. In the Student's case, the joint default probability is

$$
F(t)=t_{R, \xi}\left(t_{\xi}^{-1}\left(F_{1}(t)\right), \ldots, t_{\xi}^{-1}\left(F_{n}(t)\right)\right)
$$

In general, assuming a copula $C$, one has

$$
F(t)=C\left(F_{1}(t), \ldots, F_{n}(t)\right)
$$

\subsection{Factor copulas, or joint default simplified}

If the number of obligors increases, the representations (2) or (4), in spite of their conceptual simplicity, can become cumbersome. Therefore, it was common practice before the finincial crisis of the last years, especially for pricing and hedging applications, which can involve more than a hundred names, to substitute the actual copula with the so called factor one, as follows.

Let us assume that the asset value has unit value at time zero and normalize the log asset value (or asset return) of firm $i$. The latter is:

$$
V_{i}^{\prime}=\frac{\ln V_{i}-\left(\mu_{i}-\sigma_{i}^{2} / 2\right) t}{\sigma_{i} \sqrt{t}}
$$


An analogous expression holds under the risk neutral, pricing measure. Assume that each log asset value in the portfolio can be factorized in a common component $Z$ and an idiosyncratic one, $\varepsilon_{i}$, as follows:

$$
V_{i}^{\prime}=\rho_{i} Z+\sqrt{1-\rho_{i}^{2}} \varepsilon_{i}
$$

where $\rho_{i} \in \mathbb{R}, Z$ and $\varepsilon_{i}, i=1, . ., n$ are independent standard Gaussian. The weighting coefficients $\rho_{i}$ and $\sqrt{1-\rho_{i}^{2}}$ are chosen so that $V_{i}^{\prime}$ is standard normal. The assumptions on the factorization are such that not only the $\log$ asset values $V_{i}^{\prime}$ s are independent, conditionally on the common factor $Z$, but also that the unconditional linear correlation coefficient between two log asset values $V_{i}^{\prime}$ and $V_{j}^{\prime}$ is $\rho_{i} \rho_{j}$. The conditional marginal default probabilities, $p_{i}^{t}(z)$, if $K_{i}^{\prime}$ is the properly normalized log liability,

$$
K_{i}^{\prime}(t):=\frac{\ln K_{i}-\left(\mu_{i}-\sigma_{i}^{2} / 2\right)}{\sigma_{i} \sqrt{t}}
$$

are easily calculated:

$$
p_{i}^{t}(z)=P\left(V_{i}^{\prime} \leq K_{i}^{\prime} \mid z\right)=\Phi\left(\frac{K_{i}^{\prime}-\rho_{i} z}{\sqrt{1-\rho_{i}^{2}}}\right)
$$

The expressions for the unconditional ones follow by simple integration over the support of the factor:

$$
F_{i}(t)=\int_{\mathbb{R}} p_{i}^{t}(z) \varphi(z) d z
$$

where $\varphi$ is the standard Gaussian density.

Taking into consideration that asset values - and therefore defaults - are conditionally independent, the conditional joint default probability is simply the product of the marginal ones:

$$
F(t \mid z)=\prod_{i=1}^{n} p_{i}^{t}(z)
$$

It can be written in copula terms using the product copula $C^{\perp}$. As the name says, the latter is simply the product of its arguments:

$$
C^{\perp}\left(u_{1}, u_{2}, . ., u_{n}\right):=u_{1} \times u_{2} \times . . \times u_{n}
$$


It follows that:

$$
F(t \mid z)=C^{\perp}\left(p_{1}^{t}(z), . ., p_{n}^{t}(z)\right)
$$

The corresponding expressions for the joint unconditional probability easily follow:

$$
F(t)=\int_{\mathbb{R}} \prod_{i=1}^{n} p_{i}^{t}(z) \varphi(z) d z=\int_{\mathbb{R}} C^{\perp}\left(p_{1}^{t}(z), . ., p_{n}^{t}(z)\right) \varphi(z) d z
$$

The technique above can be extended beyond the Gaussian case. In general, if the common factor $Z$ has a density $f(z)$ on the real line $\mathbb{R}$, it follows from the definition of conditional probability that the marginal (unconditional) default probabilities can be written as

$$
F_{i}(t)=\int_{\mathbb{R}} p_{i}^{t}(z) f(z) d z
$$

The joint unconditional probabilities can be represented through the (conditional) product copula $C^{\perp}$, as desired:

$$
F(t)=\int_{\mathbb{R}} \prod_{i=1}^{n} p_{i}^{t}(z) f(z) d z=\int_{\mathbb{R}} C^{\perp}\left(p_{1}^{t}(z), . ., p_{n}^{t}(z)\right) f(z) d z
$$

\subsection{Shortcomings}

The traditional, Merton's type model tends to produce vanishing spreads at very short maturities. Generally, it underestimates the actual ones.

The model shows also poor fit over longer maturities, with overestimate of risky debt and underestimate of low-risk one. This bias has been documented by Eom, Helwege, Huang (2004) and was well known, before the crisis, both in the literature and in the practice.

Merton's model has been extended in a number of ways. One main extension consisted in including coupons on debt or debt covenants. This made default before debt-expiry possible. Another important extension consisted in including (riskless) stochastic interest rates. The extensions are well beyond the scope of this 
paper. However, once compared with actual spread data, the extended Merton's models still provide credit spreads well below the actual ones for short maturities and - even for longer maturities high ratings; well above the actual ones for longer maturities and low ratings (see Eom, Helwege, Huang (2004)).

The main reason behind the scarce consistency of Merton's model with market data is the assumption that the firm value follows a continuous process. This assumption, which is preserved by the extensions - be them through coupons, covenants or stochastic rates - makes the asset value behavior not totally unpredictable. Intuitively, no surprise can occur. As a consequence, spreads in Merton's model are generally too low. They become too high when volatility is overestimated, namely in the long run and for low credit ratings. A model is needed which does capture the actual dispersion - or volatility - by capturing the possible information arrivals, including surprises or jumps, appropriately.

\section{An alternative, business-time based model}

The alternative model we are going to present is based on the distinction between calendar and business time. This idea goes back to Clark (1973) and has been developed, for instance, by Geman, Madan and Yor (2001), Geman (2005). The idea is that business - or transaction - time runs faster than calendar time when the market is very active, trade is high, a lot of information arrives and consequently asset prices are quite volatile. It runs at a slower pace than calendar time when few information arrives and volatility is low.

The aim of adopting the previous distinction in credit risk models is that of providing both a better theoretical framework and a better empirical fit.

There is an economic rationale for our choice. The businesstime models can be written as time-changed Brownian motions. This means that prices are diffusions in business time, not in calendar time. The pace at which business time runs depends of in- 
formation and/or trade. Time in quiet trade periods does not run as in active - or hypersensitive - periods as a boom or a crisis, when everyone keeps alert, ready to buy or sell. The distinction between the two times is alike a stochastic $\operatorname{clock}^{3}$. Asset prices are not any more diffusions, once the stochastic time change has been accounted for.

The mathematical and statistical rationale is the need for asset models able to present jumps. As soon as they present jumps at the trajectorial level they will present skewness, kurtosis and other deviations from normality at the distributional level. Even apart from the credit domain, we know that in modern markets equity prices - which are a component of firm's assets - present very often deviations from normality. This is a further motivation for adopting business-time based models, such as the so called Lévy models (of the pure jump type) we are going to focus on. Only sudden jumps to default can overcome the lack of total unpredictability of default of diffusive models.

In practice, in Lévy models (of the pure jump type) the shift from the traditional, Merton's alike model is accomplished by making $V^{\prime}$ a pure jump process. If the time change has value $G_{t}$ at time $t$, the $\log$ return in calendar-time is:

$$
V_{i}^{\prime}(t)=\theta_{i} G_{t}+\sigma_{i} W_{G_{t}}^{(i)}, \quad t \geq 0 .
$$

with $\theta_{i} \in \mathbb{R}, \sigma_{i}>0$ and $W_{t}^{(i)}$ a time- $t$ Brownian motion typical of firm $i$.

In the credit domani, this device was first adopted, as far as we know, by Madan (2000). He specified the time change to be of the gamma type with parameter $\nu$. By so doing, he got the so called Variance Gamma (VG) process for log asset prices, which had been studied in Madan and Seneta (1990). As in the Merton's case, it is quite easy to shift to the risk neutral measure, by adding to the asset value the component $m_{i} t$, where

$$
m_{i}=r+\nu^{-1} \log \left(1-\frac{1}{2} \sigma_{i}^{2} \nu-\theta_{i} \nu\right) .
$$

\footnotetext{
${ }^{3}$ It cannot be a deterministic change of time, otherwise no jumps could occur. See Geman et al. (2005) on tis matter.
} 
This is the choice we are going to adopt in the sequel. In principle, a number of more sophisticated time changes could be adopted (see for instance Luciano and Semeraro (2010a,b,c)). For the present purpose, however, we will see that already the seminal one is enough to overcome the main shortcomings of the traditional models.

\subsection{Single and joint defaults}

Given the choice of the Gamma time change, we obtain what we call a VG-Merton's model.

Based on (10), the marginal conditional default probability of firm $i$, having denoted as $v_{i}$ the $\log$ debt value, $\ln K_{i}$, becomes

$$
p_{i}^{1}(z)=\Phi\left(\frac{v_{i}-m_{i}-\theta_{i} z}{\sigma_{i} \sqrt{z}}\right),
$$

Its unconditional version is

$$
F_{i}(1)=\int_{0}^{+\infty} p_{i}^{1}(z) \frac{\nu^{-1 / \nu}}{\Gamma(1 / \nu)} z^{\frac{1}{\nu}-1} \exp (-z / \nu) \mathrm{d} z
$$

since

$$
f(z)=\frac{\nu^{-1 / \nu}}{\Gamma(1 / \nu)} z^{\frac{1}{\nu}-1} \exp (-z / \nu)
$$

is the density of the gamma time change, with $\Gamma(1 / \nu)$ the gamma function.

Based on section 2.3, the joint conditional probability when $Z=$ $z$ is simply

$$
\prod_{i=1}^{2} \Phi\left(\frac{v_{i}-m_{i}-\theta_{i} z}{\sigma_{i} \sqrt{z}}\right)
$$

the joint unconditional probability, since conditional independency holds, can be written via the factor copula:

$$
F(1)=\int_{0}^{+\infty} \prod_{i=1}^{2} \Phi\left(\frac{v_{i}-m_{i}-\theta_{i} z}{\sigma_{i} \sqrt{z}}\right) \frac{\nu^{-1 / \nu}}{\Gamma(1 / \nu)} z^{\frac{1}{\nu}-1} \exp (-z / \nu) \mathrm{d} z
$$




\section{Marginal calibration: a story of suc- cess}

This section contains the results of an empirical test of the VG univariate credit model, developed by Fiorani et al. (2010). The objective of the empirical investigation was - as usual - to apply the model to real price data and to determine the performance of the theoretical suggestion behind it. In order to reach the aim, we

- Use a subsample of USA spread data to calibrate the VG parameters $\sigma, \nu$ and $\theta$ of a number of firms

- Use the calibrated parameters to forecast their credit spreads

- Compare the forecasted spreads and the actual ones (which form the remaining part of the spread sample) to assess the quality of the theoretical credit model

\subsection{Calibration}

The data we used were as follows.

- The initial calibration was done using one month of data on all the components of the Dow Jones investment grade Credit Default Swap (CDS) index (CDX NA IG) and high yield index (CDX NA $\mathrm{HY}$ ), for a total of 225 names.

○ We had a total of approximately 18,700 credit spreads. This makes our test one of the most extensive empirical test of credit risk in pure jump structural models presented in the literature to date.

○ We considered two possible CDS maturities, namely 5 years and 10 years, so as to get "time-independent" or "time-robust" conclusions.

Overall, the statistics of our spread were 


\begin{tabular}{|c|c|c|}
\hline \multicolumn{3}{|c|}{ Distribution of Average Spreads } \\
\hline \multicolumn{3}{|c|}{ Whole Sample } \\
\hline percentiles (bp) & 5 year spreads & 10 year spreads \\
\hline $5 \%$ & 23.273 & 37.227 \\
\hline $25 \%$ & 39.205 & 56.227 \\
\hline $50 \%$ & 87.045 & 101.955 \\
\hline $75 \%$ & 240.284 & 233.477 \\
\hline $95 \%$ & 556.293 & 625.205 \\
\hline mean & .019 & .020 \\
\hline st.deviation & .0310 & .029 \\
\hline skewness & 4.560 & 4.249 \\
\hline kurtosis & 28.234 & 24.608 \\
\hline
\end{tabular}

Other inputs were: the riskless rate $r$, which we took from the appropriate US swap curve; the leverage ratio $V_{i} / F_{i}$, which we took, firm by firm, from balance sheet data; the payout rate (Weighted average of debt and equity payout) $q_{i}$, which we obtained by a nameby-name elaboration on Bloomberg's data. Last but not least, we needed the recovery rate, which we got in Fiorani et al. (2010), using name-by-name data.

The calibration methodology we used was as follows.

- We minimized the sum of squared errors between theoretical and actual spreads over the in-sample (or the first part of the sample) period

- We did two separate calibrations for each firm; one for 5 year and one for 10 year spreads

Let us drop the dependence on the firm name, $i$, for simplicity. We knew that the theoretical spread was proportional to the price of a put option on a VG underlying, which depends on the initial firm value $V(0)$, the face value of debt $K$, the riskless interest rate $r$, the payout ratio $q$, expiration $t$, as well as on the firm's parameters . Let the put value be $\operatorname{VGP}(V, K, \sigma, \nu, \theta, r, q, t)$. Fiorani et al. show 
that

$$
\begin{aligned}
& V G P=V(0) \exp (-q t)\left[\Psi\left(k(d) \sqrt{\frac{1-c_{1}}{\alpha}},(\beta+s) \sqrt{\frac{\alpha}{1-c_{1}}}, \frac{t}{\alpha}\right)-1\right]+ \\
& -K \exp (-r t)\left[\Psi\left(k(d) \sqrt{\frac{1-c_{2}}{\alpha}}, \beta \sqrt{\frac{\alpha}{1-c_{2}}}, \frac{t}{\alpha}\right)-1\right]
\end{aligned}
$$

where $\alpha \doteq 1 / v, d$ is the firm's quasi-leverage ratio:

$$
d:=\frac{K \exp (-(r-q) t)}{V(0)},
$$

the function $k(d)$ is defined as

$$
\begin{aligned}
& k(d):=\frac{1}{s}\left[\ln \left(\frac{1}{d}\right)+\frac{t}{\alpha} \ln \left(\frac{1-c_{1}}{1-c_{2}}\right)\right], \\
& c_{1}:=\frac{\alpha(\beta+s)^{2}}{2} ; \\
& c_{2}:=\frac{\alpha \beta^{2}}{2} \\
& \beta:=-\theta / \sigma^{2},
\end{aligned}
$$

\begin{tabular}{|c|c|c|}
\hline \multicolumn{3}{|c|}{ VG-Merton model calibrated parameters: $\sigma$} \\
\hline & 5 year horizon & 10 year horizon \\
\hline number of names excluded & 3 & 3 \\
\hline mean & .280 & .412 \\
\hline st.deviation & .387 & .533 \\
\hline \multicolumn{3}{|c|}{ VG-Merton model calibrated parameters: $\nu$} \\
\hline & 5 year horizon & 10 year horizon \\
\hline number of names excluded & 3 & 3 \\
\hline mean & .389 & .296 \\
\hline st.deviation & .398 & .208 \\
\hline
\end{tabular}

and the function $\Psi$ can be obtained from the Hypergeometric function of two variables and the Bessel function of the second type

The results we got are in the next tables. 


\begin{tabular}{|l|l|l|}
\hline \multicolumn{3}{|c|}{ VG-Merton model calibrated parameters: $\theta$} \\
\hline & 5 year horizon & 10 year horizon \\
\hline number of names excluded & 3 & 3 \\
\hline mean & -.223 & -.111 \\
\hline st.deviation & .290 & .298 \\
\hline
\end{tabular}

\subsection{Forecast and comparison}

Using the calibrated parameters, we forecasted the spreads for the high yield (HY) and investment grade (IG) firms over the successive months. We then compared them to the observed spreads. The analysis of the errors between forecasted and actual spreads was comprehensive of three types of error: Average Daily Error (ADE), Percentage Pricing Error (\%PE), Average Percentage Error in Absolute Value (\%APE). The ADE for instance is

$$
A D E=\sqrt{\frac{\sum_{u=1}^{M}\left(\left.s\right|_{o b}(u)-\left.s^{*}\right|_{t h}(u)\right)^{2}}{M}}
$$

where $\left.s\right|_{o b}(u)$ and $\left.s^{*}\right|_{t h}(u)$ are respectively the observed and theoretical (or forecasted) spread in day $u$ for a specific name or firm, and the sum is extended over $M$ days.

Here are the results in Fiorani et al. (2010) over five years:

\begin{tabular}{|l|l|l|l|}
\hline \multicolumn{4}{|c|}{ Pricing errors from 5-year CDS's } \\
\hline percentiles & ADEx10000 (bp) & PE & APE \\
\hline $5 \%$ & 1.793 & .397 & .030 \\
\hline $10 \%$ & 2.696 & .282 & .051 \\
\hline $25 \%$ & 4.152 & .148 & .071 \\
\hline $50 \%$ & 11.331 & .067 & .100 \\
\hline $75 \%$ & 22.716 & .002 & .194 \\
\hline $90 \%$ & 41.33 & .112 & .295 \\
\hline $95 \%$ & 66.758 & .183 & .425 \\
\hline mean & 26 & .075 & .150 \\
\hline st. deviation & 70 & .190 & .143 \\
\hline
\end{tabular}


Similar results hold over 10 years. Overall, the errors are extremely low. This signals the good quality of the prediction. In particular, the ADE median is just above 10 bps for both 5 and 10 year CDS's, the mean is 26 bps and 47 bps for 5 and 10 year CDS

\subsection{Comparison with Merton's Model and sim- ilars}

We compared the above performance with the results for the Merton's model in Eom, Helwege and Huang (2004). We focused on the behavior of the previous model first in terms of under/overprediction, then in terms of bias.

For the prediction, we can examine the following table:

$\begin{array}{|cccc|} & \text { Merton's } & \text { VG-from 5 years } & \text { VG-from 10 years } \\ \text { Mean \%PE } & -50.4 \% & 7.5 \% & 6.6 \% \\ \text { sd \%PE } & 72 \% & 19 \% & 17 \% \\ \text { Mean \%APE } & 78 \% & 15 \% & 14 \% \\ \text { sd \%APE } & 40 \% & 14 \% & 13 \%\end{array}$

where sd stands for standard deviation.

Merton's model tends to underestimate the spreads. The underestimation disappears when using VG. Merton's mean errors and their sds are much larger then the ones produced by VG. Among the models tested by Eom, Helwege and Huang, the best performing model was Geske's model with a mean \%APE of $65.7 \%$ and sd $28.3 \%$, much worse than ours.

We then considered the bias. Indeed, as we said above, Merton's model severely underestimates short term spreads. To adjust for Merton's model main problem, alternative models severely overestimate the high-risk spreads and underestimate low-risk ones. Does the VG model suffer from the same bias? In order to answer, we separated our dataset in 4 groups: HY (riskier) versus IG (safer) and 5 year versus 10 year spreads.

Consider for instance the 5-year IG CDS. 


\begin{tabular}{|l|l|l|l|}
\hline \multicolumn{4}{|c|}{ Pricing errors from 5-year IG CDS's } \\
\hline percentiles & ADEx10000 (bp) & PE & APE \\
\hline $5 \%$ & 1.460 & -.172 & .040 \\
\hline $10 \%$ & 2.007 & -.120 & .059 \\
\hline $25 \%$ & 3.054 & -.044 & .078 \\
\hline $50 \%$ & 5.053 & .065 & .106 \\
\hline $75 \%$ & 12.700 & .140 & .194 \\
\hline $90 \%$ & 18.868 & .342 & .383 \\
\hline $95 \%$ & 22.716 & .457 & .465 \\
\hline mean & 8.3 & .075 & .171 \\
\hline st. deviation & 7.4 & .227 & .172 \\
\hline
\end{tabular}

The \%PE mean is $7.5 \%$, low and positive. This means that there is low overpricing. The APE mean is low too. This means that there is no compensation. So there is no bias in this case.

Fiorani et al. consider also the other groups.

- IG and HY errors are very close to each other, for 5 and 10 years.

- Errors have the same signs at the same percentile (this is shown by the \% PE)

- The ADE shows an higher error in terms of basis points, because HY's spreads are much higher than IG ones. When we scale by the size of the error, such as in the \%PE and \%APE, IG and $\mathrm{HY}$ error sizes are very close.

The conclusions of the univariate analysis then were:

- The Merton's VG model presents small errors both in absolute terms and in comparison to Merton's model and to other structural models;

- Merton's underprediction is amended by its VG version, without introducing other biases (such as overprediction of HY CDS spreads, which is typical of the refinements of Merton's model)

- The VG-Merton's model performs well for both HY and IG spreads and for both 5 and 10 year spreads. 


\section{Joint calibration and factor default probabilities}

Once VG is shown to be superior at the single-default level, one can use it for joint default assessment.

As an example, we report the results obtained in Luciano (2006). Here a sample of five names was selected: Autozone, Ford, Kraft, Walt Disney, Whirlpool. The parameters of their asset values under the VG hypothesis had been derived (from CDS prices) in Luciano and Schoutens (2006). Dependence was computed under the assumption of a unique change of time for all the firms in the sample. This simplified the calibration, because the computation of the assets'correlation or dependence was not required. However, the methodology can be generalized ${ }^{4}$.

The next two tables present respectively their calibrated joint probabilities under the traditional Merton's model and its VG extension

\begin{tabular}{|c|c|c|c|c|c|}
\hline \multicolumn{6}{|c|}{ Joint default probabilities, Merton model } \\
\hline & Autozone & Ford & Kraft & Walt Disney & Whirlpool \\
\hline Autozone & & 6.065 & 0.030 & 0.053 & 0.508 \\
\hline Ford & 6.065 & & 0.080 & 0.143 & 1.356 \\
\hline Kraft & 0.030 & 0.080 & & 0.001 & 0.007 \\
\hline Walt Disney & 0.053 & 0.143 & 0.001 & & 0.012 \\
\hline Whirlpool & 0.508 & 1.356 & 0.007 & 0.012 & \\
\hline
\end{tabular}

\begin{tabular}{|c|c|c|c|c|c|}
\hline \multicolumn{6}{|c|}{ Joint default probabilities, VG model } \\
\hline & Autozone & Ford & Kraft & Walt Disney & Whirlpool \\
\hline Autozone & & 2.421 & 0.599 & 0.712 & 1.215 \\
\hline Ford & 2.421 & & 0.966 & 1.149 & 2.035 \\
\hline Kraft & 0.599 & 90.966 & & 0.350 & 0.565 \\
\hline Walt Disney & 0.712 & 21.149 & 0.350 & & 0.667 \\
\hline Whirlpool & 1.215 & 52.035 & 0.565 & 0.667 & \\
\hline
\end{tabular}

Intuition suggests that neglecting jumps - or fat tails or sudden information arrival and the volatility it creates - leads to underestimating joint default probabilities. The percentage differences in

\footnotetext{
${ }^{4}$ Luciano and Semeraro (2010b,c) extend the theoretical model wrt this restriction.
} 
the Merton's and pure jump default probabilities visualized below confirm the intuition. They also point out the exceptions: this is important, in that the differences rationalize our financial sensation showing that it does not hold unconditionally.

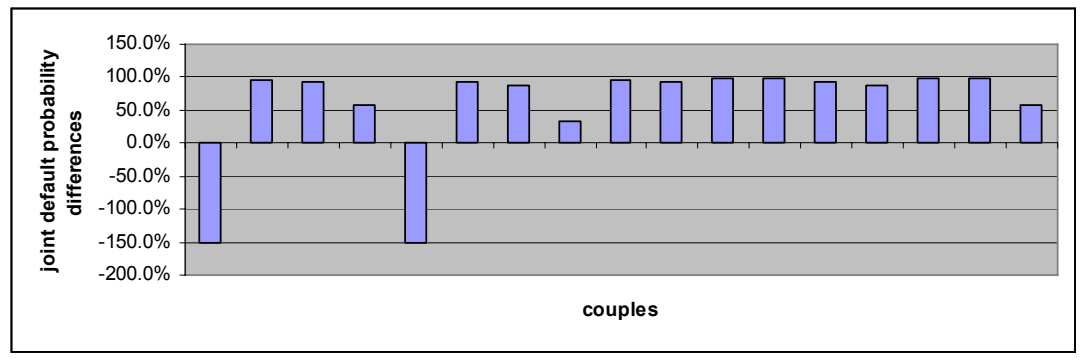

The previous table is important in that respect. We know that standard models were poor predictors of the recent financial crisis, since they underestimated joint default, as the above Merton model does in the example. The previous table shows that models able to overcome such problem - at the joint, not only the single level exist.

\section{Conclusions}

We have presented an innovative model for credit risk evaluation, based on Madan (2000), Luciano and Semeraro (2010b,c). We have strengthened the theoretical model presentation with an assessment of its empirical performance, based on Fiorani, Luciano, Semeraro (2010), Luciano (2006).

The model seems to perform well on market data. This happens because - by construction - it captures jumps - i.e. sudden changes in asset values. Based on the previous analysis, we dare to suggest that its use could complement future analyses of credit risk, in turbulent times such as the present one, when sudden information arrivals are - so to say - everyday life.

Our model however is not the only one in this strand of literature: for an account of the state of the art, see Cariboni and Schoutens (2009). 


\section{References}

Cariboni, J. and W. Schoutens, 2009, Lévy Processes in Credit Risk, (Wiley Finance, Padstow, UK).

Cherubini U., E. Luciano, and W. Vecchiato, 2006, Copula Methods in Finance, (John Wiley, Padstow, UK).

Clark, P. K., 1973, A subordinated stochastic process model with finite variance for speculative prices, Econometrica, 41, 135155 .

Eom, Y. H., Helwege, J., and J. Huang, 2004, Structural Models of Corporate Bond Pricing: an Empirical Analysis, The Review of Financial Studies, 17, 499-544.

Fiorani, F., E. Luciano and P. Semeraro, 2010, Single and joint default in a structural model with purely discontinuous assets, Quantitative Finance, 10 (3), pp. 249-264.

Geman, H., Madan, D. B. and Yor, M., 2001, Time changes for Lévy processes, Mathematical Finance, 11, 79-96.

Geman, H. 2005, From measure changes to time changes in asset pricing, Journal of Banking and Finance, 29, 2701-22.

Luciano, E., 2006, Copulas and dependence models in credit risk: diffusions versus jumps, Statistica Applicata, 18, 573-588.

Luciano, E. and W. Schoutens, 2006, A Multivariate JumpDriven Financial Asset Model, Quantitative Finance, 6, 385-402.

Luciano E., and P. Semeraro, 2010a, Multivariate Variance Gamma and Gaussian dependence: a study with copulas, in Mathematical and Statistical Methods for Actuarial Sciences and Finance, ed. by M. Corazza and R. Pizzi, (Springer Verlag).

Luciano, E., and P. Semeraro, 2010b, A Generalized Normal Mean Variance Mixture for Return Processes in Finance, International Journal of Theoretical and Applied Finance, 13 (3), May 2010 .

Luciano, E. and P. Semeraro, 2010c, Multivariate Time Changes for Lévy Asset Models: characterization and calibration, Journal of Computational and Applied Mathematics, 233, 1937-53.

Madan, D.B. and E. Seneta, 1990, The Variance Gamma (V.G.) Model for Share Market Returns, Journal of Business, 63, 511-524.

Madan, D.B., 2000, Pricing the Risks of default, working paper, 
University of Maryland.

Merton, R., 1974, On the Pricing of Corporate Debt: the Risk Structure of Interest Rates, Journal of Finance, 29, 449-470.

Nelsen R. B., 1998, An Introduction to Copulas, (Springer Verlag, New York, NY). 